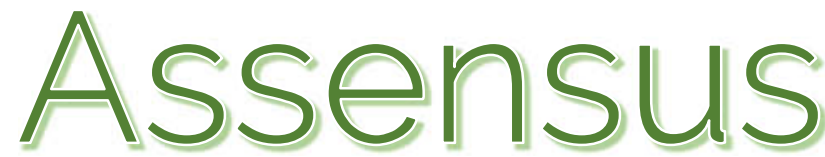

Revista de Investigación educativa y pedagógica

\title{
Caracterización de prácticas ambientales en relación con el manejo de recursos y cuidado del Entorno Escolar Rural
}

\section{Characterization of environmental practices in relation to resource management and care of the Rural School Environment}

\author{
Gregorio Gabriel \\ López López \\ Universidad de Córdoba, \\ Colombia \\ gregorio0605@hotmail.com
}

\author{
Gabriel Ángel \\ Julio Galvis \\ Universidad de Córdoba \\ Colombia \\ gajuliogalvis@gmail.com
}

\author{
Nabi del Socorro \\ Pérez Vásquez \\ Universidad de Córdoba \\ Colombia \\ ndperezvasquez@correo.unicordoba.edu.co
}

\begin{abstract}
Resumen
Palabras claves:

Educación

ambiental, prácticas

ambientales,

comportamientos, actitudes, hábitos.

El artículo muestra resultados de una investigación cuyo propósito fue la caracterización de las prácticas ambientales en estudiantes de básica secundaria de la zona rural del municipio de Montería, en relación con el manejo de los recursos agua, energía, residuos sólidos y cuidado del entorno escolar. Con un enfoque cualitativo mediante la investigación acción, desde el paradigma sociocrítico, que acerca al investigador a la problemática detectada apoyándose en actores claves para transformar la realidad a partir de la interpretación de resultados, se utilizan técnicas como la encuesta aplicada a estudiantes y docentes, observación participante, y entrevistas semiestructuradas a grupos focales de estudiantes con las que se logró recopilar información sobre los puntos de vista, modos de pensar, de hacer, de actuar y las percepciones de los estudiantes sobre la trasformación de las prácticas ambientales. Se evidenciaron prácticas ambientales inadecuadas, descuido por el entorno escolar, bajo nivel de conocimiento ambiental. Al caracterizar las prácticas ambientales se evidenciaron acciones negativas o desfavorables en la mayoría de los estudiantes, quienes hacen manejo inadecuado del agua al usar las unidades sanitarias, al tomar agua en las piletas y lavarse las manos. El manejo de residuos sólidos es el principal problema ambiental de la institución, denotando prácticas inadecuadas hacia el ambiente.
\end{abstract}

Recepción: May 12 de 2020 | Aceptación: Jun 15 de 2020 | Publicación: Dic 15 de 2020 


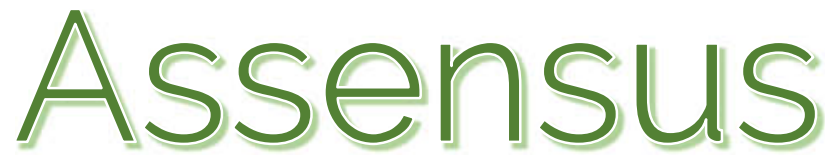

Revista de Investigación educativa y pedagógica

\begin{tabular}{ll}
\hline & Abstract \\
& The article shows results of an investigation whose purpose was \\
the characterization of environmental practices in the students of \\
secondary education of the Educational Institution Morindó Santa \\
Fé, located in the rural area of left bank of the municipality of \\
Monteria, in relation to water management, energy, solid waste \\
resources and care of the school environment. All this thanks to \\
a qualitative approach completed by action research, from the \\
sociocritical paradigm, which links the researcher with the \\
identified problem, with the support of the main actors to change \\
reality based on the interpretation of the results obtained from \\
the surveys. applied to students and teachers, observation guides \\
and structured semi-interviews with focus groups that managed \\
to collect information on the points of view, ways of thinking, of \\
doing, acting and perceptions of students about the \\
transformation of environmental practices.. Then la institution \\
was evidenced of inadequate environmental practices, neglected \\
by the school environment, low level of environmental knowledge. \\
By typifying environmental practices, most students attempted to \\
disapprove of the actions. For example, inadequate water \\
management when using toilets. In addition, they wash their \\
hands with recycled water in containers. In fact, solid waste \\
management is considered the main environmental problem in \\
this place. Garbage is thrown anywere, mainly during cultural \\
and civic events. The use of containers is almost useless, \\
denoting inappropriate practices towards the environment.
\end{tabular}

attitudes, habits.

Received: May 12, 2020 | Accepted: Jun 15, 2020 | Published: Dec 15, 2020 


\section{Introducción}

La investigación nace de la lectura del contexto escolar donde, además de caracterizar las prácticas ambientales en relación con el manejo de los recursos agua y energía, de los residuos sólidos y el cuidado de las áreas comunes, se desarrolló una evaluación formativa que develó la necesidad de transformarlas desarrollándose acciones educativas a partir de estrategias pedagógicas para minimizar las prácticas desfavorables y potenciar las buenas como aporte fundamental del método de investigación acción y del enfoque sociocrítico, sin embargo en el documento que se despliega a continuación se presentan los resultados de la caracterización.

La sociedad moderna se enfrenta a distintos desafíos ambientales relacionados con la crisis ambiental global. Desde los inicios de la humanidad el hombre ha tenido una estrecha relación con el medio en el que vive, "aprovechando lo que la tierra le provee e interactuando con ella, incluso sintiéndose con pleno derecho para explotar sus bienes y recursos, lo que provocó una aceleración de los procesos de degradación del medio ambiente". (CastilloRetamal y Cordero-Tapia, 2019, p. 2); esto ha llevado a la necesidad de repensar las formas de interacción de la humanidad con el ambiente, hacia nuevas maneras de ser y estar en el planeta, en relación con el otro humano y lo otro que no es humano, "es preciso reorientar la forma de relacionarnos entre nosotros y con el resto de la naturaleza, para poner las bases de un presente sostenible y satisfactorio para el conjunto de los seres humanos". (Vilches y Pérez, 2016. p.405); por tanto, la escuela tiene la tarea de formar ciudadanos responsables y comprometidos con el ambiente, reconocerlo desde un enfoque sistémico e integral y, además, en la motivación para que cada uno de los habitantes del mundo se sensibilice y actué de forma armoniosa con él.

En este sentido, la educación ambiental es fundamental al propiciar desde las escuelas y los diferentes centros educativos acciones para favorecer en las nuevas generaciones el desarrollo de hábitos amigables con el ambiente, a fin de modificar las conductas erróneas. En todo caso, "el fomento de la responsabilidad requiere de un fuerte impulso de la educación ambiental (EA) en el ámbito formal y no formal que favorezca y promueva buenas prácticas ambientales" (Castillo y Fuentealba, 2014, p. 8). Se hace necesario que en las instituciones educativas como espacios de formación importantes en la apropiación de conocimientos en educación ambiental coadyuven a transformar la cultura de los estudiantes, desde un saber ambiental, que conduzca a cambios en las formas de vida y pensamiento, hacia una nueva conducta social e individual, de la mano de una reestructuración de la economía y la sociedad. Una formación ambiental que genere conciencia determine comportamientos, actitudes, hábitos, decisiones, como lo reconocen Gomera, et al., (2013), al señalar que una conciencia ambiental integral establece una conexión entre varios constructos psicológicos (conocimientos, información, normas, valores, actitudes, creencias) que desencadena 
comportamientos que posibilitan convivir con el entorno, preservarlo y transformarlo en función de las propias necesidades, sin comprometer con ello la posibilidad de las generaciones futuras; uno de los propósitos de la EA es que la comunidad comprenda la naturaleza compleja del ambiente y, a partir de ello, adquirir "conocimientos, valores y habilidades prácticas para participar en la prevención y solución de problemas ambientales".(Calderón, et al., 2011, p. 21); por lo que analizar, diagnosticar y desarrollar herramientas para potenciar buenas prácticas ambientales es un paso determinante a la hora de diseñar e implementar planes y programas eficientes de educación ambiental. De ahí la necesidad de caracterizar las prácticas ambientales (PA), en tanto, al analizar "las actitudes y percepciones ambientales de los estudiantes se puede conocer el tipo de educación ambiental que reciben" (Fuentealba, 2018, p, 161).

Para contextualizar el problema, se destaca que mediante observaciones efectuadas a la comunidad y al entorno familiar de los estudiantes de la IE Morindó Santa Fé, se evidenció que no se preocupan por generar cultura ambiental en sus casas, realizan acciones diarias que así lo demuestran, no hacen adecuada separación de residuos; es más habitualmente los queman a cielo abierto, incluso la hojarasca y otros residuos vegetales, asimismo botan parte de sus desperdicios a la quebrada Morindó, el uso del agua es inadecuado y existe muy poca reflexión acerca de las actuaciones individuales que ejecutan diariamente, comportamientos que se reflejan en la institución donde además reconocen que existe un desconocimiento ambiental, sus conductas son poco amigables con el entorno, se encontró falta de cooperación, de solidaridad, de sentido de pertenencia institucional, lo que da cuenta de prácticas ambientales inadecuadas o negativas que si bien fueron identificadas a través de observaciones preliminares, no se han sistematizado en un estudio que revele como están influyendo los comportamientos, hábitos y actitudes, frente al cuidado, conservación, uso racional de los recursos y de su ambiente natural en sus prácticas ambientales, así como en su proceso formativo, lo que revela la importancia que tiene desarrollar una educación ambiental que los lleve a transformar sus prácticas ambientales.

\section{Referentes teóricos}

\section{Acciones ambientales, referentes de la practicas cotidianas}

Las prácticas ambientales (PA), se relacionan con "acciones que garantizan la reducción del impacto negativo en el ambiente” (Secretaría de Ambiente. Alcaldía de Quito. 2018, p.3), además, están en concordancia con "comportamientos favorables hacia el ambiente; que buscan conscientemente proteger, preservar y/o minimizar los impactos negativos sobre el medio ambiente". (Rivera-Torres y Garcés-Ayerbe (2018 p. 60). 
En las prácticas se interrelacionan factores antecedentes y consecuentes, que crean un campo de elementos que correlacionan con la presencia de ciertos tipos de actividades dentro de los espacios individuales y colectivos. Estos campos de interacción involucran consecuencias sociales y naturales en las interrelaciones con el ambiente a nivel próximo y distal, las cuales ocurren como parte de unas condiciones físicas y sociales del contexto que llevan a patrones de comportamientos asociados al pensar, sentir, reaccionar físicamente y actuar en determinada situación (Sandoval, 2012). Por su parte, "las buenas prácticas, aluden a comportamientos, procedimientos o hábitos deseables que se deben llevar a término para conseguir determinados objetivos con la mayor eficacia y eficiencia". (Universidad de Cádiz, 2010, p.5). En Colombia desde hace más de dos décadas desde el Ministerio de Medio Ambiente y Desarrollo Sostenible se ha desarrollado documentos y guías para promover el desarrollo sostenible en sectores de producción nacional. "Las guías ambientales corresponden al concepto mundial de Buenas Prácticas Ambientales o Mejores Prácticas Ambientales, instrumentos técnicos que consolidan modelos o esquemas para el mejoramiento de la gestión, manejo y desempeño ambiental de los sectores productivos" (García y Restrepo 2015 p. 262).

De hecho, son pocos los trabajos en el ámbito educativo. Reafirmando lo anterior, la Presidencia de la República de Colombia precisa las buenas prácticas ambientales como "un conjunto de acciones sencillas que implican un cambio de actitud y de comportamiento en nuestras actividades diarias, promoviendo una relación amigable con el ambiente" (Presidencia de la República de Colombia, 2016, p. 3). Según lo expresado en el manual de buenas prácticas ambientales del Senado de la República de Colombia Dirección General Administrativa (2011) "a través de la implementación de las Prácticas Ambientales se tiene la posibilidad de reducir el impacto ambiental negativo generado por las actividades de cada persona de manera individual" (p. 5). En este orden de ideas, las prácticas están en relación con "las actitudes ambientales que son sentimientos favorables o desfavorables que se tienen hacia alguna característica del ambiente físico o hacia un problema relacionado con él” (Galli, et al., 2013, p.462). Además, "generan comportamientos o cambios para alcanzar propósitos determinados, beneficios o transformaciones...la formación, las creencias, las costumbres, la cultura, el sentido de pertenencia, las condiciones de vida de la sociedad, las normas, el fortalecimiento institucional y la ética generan determinadas prácticas que tienen efectos consecuentes en el medio ambiente, alterando las condiciones de vida, las prácticas ambientales determinan condiciones y calidad de vida de las personas en las instituciones y en la sociedad" (García y Restrepo 2015 p. 265).

\section{Educación ambiental, desde lo axiológico a la acción}

Desde la Carta de Belgrado (1975) se enunció una educación ambiental que tuviera como uno de sus propósitos lograr la concienciación de los problemas cotidianos; comprender críticamente la realidad a través del conocimiento, impulsando valores sociales y apuntar a la 
participación activa de los actores sociales en la protección y mejoramiento del ambiente. De acuerdo con Flórez (2013) la educación ambiental "tiene como objeto de estudio las relaciones del ser humano con el medio ambiente, para contribuir a transformar esta relación, incidiendo en la construcción de hábitos, actitudes, valores y comportamientos, proambientales" (p.96). De acuerdo con Novo (2009), es esencial que "la educación ambiental se mueva en el plano axiológico, en primer lugar, para desactivar algunos de los señuelos que, en nuestras sociedades, ofrecen un supuesto "progreso" a base de tener más y consumir más" (p.123), siendo la herramienta para que todas las personas adquieran conciencia de la importancia de preservar su entorno y sean capaces de realizar cambios en sus valores, conducta y estilos de vida, así como ampliar sus conocimientos para impulsarlos a la acción mediante la prevención y mitigación de los problemas existentes y futuros (Severiche-Sierra, Gómez-Bustamante y Jaimes-Morales, 2016 p. 269).

Por ello, la educación ambiental debe ser un proceso activo, reflexivo y de interés colectivo de la comunidad educativa reflejado en las buenas prácticas ambientales, como medio para el cuidado, y la conservación de los recursos del entorno en relación con tres componentes básicos: actitudes, comportamientos y hábitos. Ahora, las actitudes son una confluencia de tres componentes, el cognitivo, que reúne información, experiencias, estereotipos y conocimientos, los cuales, pueden ser juicios positivos o negativos; el afectivo, que combina sentimientos, emociones, valores, satisfacciones y aversiones y el comportamental, mediado por las habilidades motoras, psíquicas, cognitivas, verbales y sociales (Cuartas-Gómez, et al., 2019, p. 2).

La formación ambiental debe llevar al sujeto a reflexionar sobre actividades cotidianas y al entendimiento de que hay que preservar el ambiente para la actual y futuras generaciones; debe generar cultura ambiental, en correspondencia a "la forma como los seres humanos se relacionan con el medio ambiente, y para comprenderla se debe comenzar por el estudio de los valores; estos, a su vez, determinan las creencias y las actitudes y, finalmente, todos son elementos que dan sentido al comportamiento ambiental (Miranda, 2013 p. 94). En este sentido, para elevar el nivel de cultura ambiental de la población, la educación ambiental se convierte en prioridad dado que a partir de ella el individuo es capaz de construir y producir conocimientos, reorientar sus valores, modificar sus comportamientos y contribuir como sujeto individual a la transformación de la realidad del medio ambiente (Ferrer, Menéndez y Gutiérrez, 2004, p. 64). Además, la cultura refleja las formas de vida de la comunidad, siendo reproductora generacional de hábitos, costumbres, tradiciones, normas y valores en ella, la educación es importante como parte de la cultura. En este sentido, se requiere una educación ambiental que brinde a cada individuo las herramientas necesarias para que actúe en forma responsable con el ambiente, con acciones proambientales voluntarias como "proceso, democrático, dinámico y participativo, para despertar en el ser humano una conciencia, que le permita identificarse con la problemática socio ambiental, tanto a nivel general, como del medio en el cual vive" (Rengifo, et al., 2012, p.4,). La educación ambiental es la herramienta 
formativa para adoptar esa cultura y propiciar cambios de actitud y aptitud y de lograr un equilibrio entre el hombre y su entorno.

En Colombia los Proyectos Ambientales Escolares (PRAE) son instrumentos de sensibilización, educación y participación que apoyan los mecanismos de gestión ambiental local con el fin de proporcionar herramientas normativas y formativas a la comunidad educativa y particular como una estrategia para el mejoramiento de los problemas ambientales de la zona y para el crecimiento social a través de propuestas que parten de los intereses y expectativas de cada individuo y colectividad. (Mora-Ortiz, 2015, p.73). Aunque la Institución Educativa Morindó Santa Fé, posee un PRAE, que se presenta a la Secretaría de Educación Municipal como un requisito, este no se dinamiza adecuadamente, sólo se ha limitado a dictar charlas con poca profundidad en el discurso, jornadas de ornato y aseo, que se hacen una vez al año, constituyéndose en activismo ambiental, dejando de lado, una formación integral basada en estructuras conceptuales y pedagógicas que conduzcan a conocimientos profundos, como lo reconoce Gutiérrez (2015), al señalar que la educación ambiental escolar, se ha relacionado con un activismo y reduccionismo en cuanto se da cumplimiento a los lineamientos estatales como el PRAE, pero de una manera no interdisciplinar, se ha convertido en una tarea específica del área de ciencias naturales con una programación que no delimita ni enfatiza en contenidos escolares que logren desarrollar en los alumnos cambios en pro del ambiente.

Los PRAEs, "en algunos casos se han convertido en activismo ecológico mediático, campañas de aseo, manejo o transformación de residuos, repetición de frases y conductas, que en muchos casos resultan insuficientes, toda vez que no generan cambios profundos en la forma de actuar, sentir y pensar de la comunidad educativa, en otras instituciones son proyectos de papel bien concebidos, pero convertidos en letra muerta, no se desarrolla una formación ambiental trascendental en la vida de los estudiantes en y fuera del aula" (PérezVásquez 2020 p.26). Desconociendo que los Proyectos Ambientales Escolares, se concibieron como un instrumento para incluir la dimensión ambiental en los Proyectos Educativos Institucionales PEI e impactar a los estudiantes con un aprendizaje significativo que contribuya al desarrollo de competencias ciudadanas y de pensamiento científico para el fortalecimiento de los procesos de gestión ambiental (Gómez, 2018, p.181)

Al hacer una revisión teórica de estudios relacionados con el tema investigativo, se destaca el trabajo de investigación elaborado por Pineda y Pinto (2018) con el objetivo de diseñar una propuesta didáctica en educación ambiental para el fortalecimiento de las buenas prácticas ambientales para el cuidado y preservación del patrimonio ambiental, social y cultural en la comunidad educativa de la Escuela Normal Superior sede Vicente de Paúl del municipio de Leticia, Amazonas, entre los resultados destaca como uno de los problemas ambientales el mal manejo del recurso agua, energía, poco cuidado de jardines, pero el que más afecta a la institución y la comunidad es manejo inadecuado de los residuos sólidos. Orbes y Delgado (2017) desarrollan una investigación con el propósito de comprender las 
concepciones y prácticas ambientales de docentes y estudiantes para fortalecer la enseñanza del ambiente en el grado 7-1 de la I.E.M. Nuestra Señora de Guadalupe, evidenciando prácticas ambientales desfavorables por parte de los estudiantes en cuanto al desperdicio de papel y energía, además de la disposición inadecuada de residuos, y el desperdicio de agua. Asimismo, Pérez (2015) con el objetivo de comprender los sentidos que la comunidad de la Institución Educativa Cámara Junior de la ciudad de Armenia (Quindío), les otorga a las prácticas ambientales actuales en relación con las problemáticas medioambientales y el desarrollo sostenible. En los resultados da cuenta de que el principal problema es el inapropiado manejo de los residuos sólidos, de igual forma, evidenció desinterés frente al significado del medio ambiente y muy pocos la relacionan con desarrollo armónico, conservación y equilibrio. Las investigaciones antes referenciadas mostraron el mal manejo de residuos sólidos como la principal problemática que afectaba el ambiente del contexto escolar por los comportamientos, hábitos y actitudes impropias de los estudiantes, condición similar al encontrado en la investigación que ocupa el presente artículo.

\section{Metodología}

Esta investigación se enmarcó en el enfoque cualitativo, permitiendo un acercamiento a la realidad social objeto de estudio. Según Souza (2009), el enfoque cualitativo, es adecuado al estudio de la historia, las relaciones, las representaciones, las creencias, las percepciones y de las opiniones, producto de las interpretaciones que los humanos hacen con relación a cómo viven, construyen sus instrumentos y así mismo, como sienten y piensan con un diseño interpretativo.

La población objeto de estudio fueron estudiantes $\left(6^{\circ}\right.$ a $\left.9^{\circ}\right)$ y 11 docentes de educación básica secundaria de la Institución Educativa Morindó Santa Fé, de Montería, Córdoba. La recolección de los datos se efectuó a través de la observación participante, implicada en la necesidad de un trabajo cuidadoso, dado que el investigador debe integrarse al grupo, comunidad o institución, una vez allí, ir haciendo una doble tarea: desempeñar algunos roles dentro del grupo, como uno más de sus miembros, a la que va recogiendo los datos que necesita para la investigación. (Sabino, 1992, p. 113). Del mismo modo se aplicó una encuesta con preguntas abiertas y cerradas tipo Likert, de acuerdo con Carabias (2002) este es el instrumento más utilizado en la evaluación de creencias, hábitos y comportamientos, en este se pide a los encuestados que "informen, mediante cuestionarios, sobre la frecuencia con que realizan un conjunto más o menos representativo de acciones, dentro de uno o varios dominios de comportamiento, actitudes o creencias" (p.148). Los datos recolectados fueron tabulados y posteriormente se hizo la triangulación con lo manifestado por docentes, estudiantes y las observaciones efectuadas, lo que permitió la interpretación de los datos. 
En este sentido, la educación ambiental debe considerar las realidades ambientales del contexto del individuo, dado que su implicación será más directa, incluyendo el sentir y la participación de los actores sociales para el desarrollo. Sobre todo, porque padres, maestros, vecinos y compañeros, comparten un sistema de reglas donde es comúnmente aceptado el no reciclaje de las basuras, el vertimiento en lugares prohibidos, el desperdicio del agua y la electricidad, la violación de normas de contaminación visual y auditiva, así como la explotación, eco degradante de los recursos naturales en diferentes sectores (Sandoval, 2012, p. 191).

\section{Resultados}

A partir de los datos obtenidos con la encuesta, la observación participante y la recolección de los testimonios de los docentes se pudo determinar que en la Institución Educativa Morindó Santa Fé predominan prácticas ambientales poco amigables. Con el propósito de apoyar la reflexión de las prácticas ambientales, se establecieron diferentes categorías de análisis: comportamientos, hábitos y actitudes ambientales para facilitar el proceso de caracterización de estas, en cuanto al ahorro de agua, de energía, manejo de residuos sólidos y cuidado de áreas comunes. En la investigación, el conocimiento fue un elemento de análisis indispensable para caracterizar las prácticas ambientales, en tanto este no es una acción, como si lo son los hábitos y comportamientos; sin embargo, puede influir de manera positiva o negativa en las actuaciones desarrolladas por los estudiantes de la institución, aunque este no sea el factor determinante de las mismas, en tanto, influyen aquí los valores, las percepciones y las actitudes que en conjunto determinan el accionar de la persona, en este caso concreto hacia el ambiente.

Al indagar sobre conocimientos ambientales los estudiantes expresaron que reciben información en educación ambiental en la escuela principalmente, aunque reconocen que son pocos los desarrollos, por ello, un número considerable reconoce que se encuentra algo informado sobre temas ambientales. A pesar de lo encontrado en sus respuestas hay que decir que sus comportamientos ambientales se alejan de esa realidad, en tanto, muestran poco respeto hacia la naturaleza, su participación en las actividades escolares es mínima. Al estar al tanto en cuestiones ambientales sus acciones humanas deben propender hacia el cuidado y preservación de la naturaleza y que estas sean muy eficaces, sobre todo que se cuente con la participación de la mayoría, y en este caso no está reflejado en las formas de actuar que se llevan a cabo en el entorno natural tales como el cuidado de la naturaleza, ahorro de agua, manejo adecuado de residuos sólidos, y el respeto por otros seres vivos, es decir, sus comportamientos, hábitos y actitudes no están en concordancia con los conocimientos que dicen poseer sobre educación ambiental. 
De hecho, cuando se les preguntó sobre el concepto de ambiente, la mayoría respondió "el ambiente es todo lo que nos rodea", otros lo definen como "componentes de la naturaleza, o la relación entre los factores bióticos y abióticos", en la primera concepción de ambiente, se considera al ser humano como centro de todo, idea dominante desde las primeras civilizaciones, una vocación fundamental, en el contexto de su realidad que muestran los signos de una mala relación ante la misma, es evidente que la mayoría de los estudiantes manejan una concepción reduccionista del ambiente; lo que implica que conciben a la naturaleza únicamente como la fuente de recursos para la humanidad, una concepción antropocéntrica de lo que es el ambiente, reflejado en el papel predominante del ser humano sobre los demás elementos que constituyen el mundo natural, en donde coexisten todos los seres vivos, viendo el ambiente desde una perspectiva utilitarista, posicionándose el ser humano como especie dominante en la tierra, controlando su uso y definiendo sus mecanismos de producción, lo que ha llevado a la crisis ambiental actual, por ello se requiere transformar esta concepción hacia una biocéntrica, donde los estudiantes reconozcan y defiendan la existencia de valores propios en los seres vivos y en el ambiente, tal como lo señala Boff (2002) al expresar que la humanidad tiene que tomar en consideración a los demás organismos de la retícula de la vida con los que la humanidad está en profunda relación de parentesco por el hecho fundamental de que la vida es una y única"(p.20).

En este orden de ideas los estudiantes consideran la naturaleza como fuente de recursos necesarios para satisfacer las necesidades e intereses, evidenciándose el desconocimiento del papel que cumplen las interrelaciones del mundo natural social y cultural. Es importante, destacar que el análisis de las relaciones e interrelaciones entre los subsistemas social, natural, cultural y de los problemas y potencialidades ambientales, sólo es posible en la medida en que se tenga una adecuada formación ambiental, por tanto, se requiere de un proceso educativo ambiental que lleve implícito asumir con compromiso la apropiación del concepto de ambiente, la producción de nuevos saberes y recuperar la función crítica, prospectiva del conocimiento.

Por otro lado, los educandos reconocieron que el principal problema ambiental de la institución es la producción de residuos, y otros asumen que es el despilfarro de agua y la falta de zonas verdes, aquí se debe reconocer que la escuela debe ser un escenario con espacios que propicien el bienestar y el deseo de estar en ella. Hoy la creciente generación de residuos, derivado de acciones humanas, se constituye en un grave problema social y ambiental que se ha convertido en una cuestión de suma importancia hacia la que se están dirigiendo políticas de intervención, información y gestión, dado que la gran acumulación de residuos puede provocar impactos negativos, referidos no solo al ámbito ambiental, sino también al económico, sanitario y social, de esta situación se puede inferir que los estudiantes aún no han tomado conciencia de los efectos de dicha problemática, lo que puede estar relacionado con el desconocimiento sobre el tema y acerca de la gestión adecuada que se 
debe hacer con los residuos; sumado a ello la falta de interés y compromiso de no arrojar basura dentro del aula y en el patio.

Uso del recurso agua. El agua es el recurso indispensable para la vida en el planeta. Todos los seres vivos necesitan de este líquido para la realización de sus procesos vitales y de múltiples actividades. Es importante que los estudiantes comprendan que el recurso hídrico garantiza la continuidad de todas las especies, que es uno de los recursos que necesita mayor cuidado, atención y preservación.

En este sentido, una media significativa de los estudiantes reconoce no hacer buen uso de este recurso o solo algunas veces; acciones que revelan falta de cultura y prácticas ambientales desfavorables, al considerar al recurso como inagotable. Lo que denota actitudes, comportamientos y hábitos inadecuados con el ambiente. Al contrastar lo anterior, con lo expresado por los docentes sobre los comportamientos y hábitos de los estudiantes a este respecto, manifestaron que la mitad de los escolares no hacen buen manejo de agua, en las actividades diarias dentro de la escuela, por ejemplo, al usar las unidades sanitarias, al tomar agua en las piletas, desperdician el líquido. Los educadores también expresaron que en algunas ocasiones se ha evidenciado la buena intención del estudiantado por el uso racional del agua pero que en la mayoría de los casos la desperdician. Al comparar lo dicho por los docentes, con las observaciones hechas se pudo notar que hay estrecha relación en los resultados de este aspecto, en tanto, la mitad de los educandos no hace uso racional del agua, lo cual demuestra malas prácticas ambientales de un alto porcentaje de escolares. Según Arreguín (1991) "el uso eficiente no sólo aporta beneficios al sistema que lo efectúa, también significa mejora para otros usuarios" (p.10).

Manejo de basuras. La sociedad siempre ha generado desechos como resultado de los procesos de producción y consumo para satisfacer sus necesidades, cuando se preguntó a los estudiantes sobre el manejo que les dan a los residuos en la escuela, el 50\% manifestó que arrojan los desechos en cualquier lugar (Gráfico 1). Prácticas ambientales desfavorables, lo que es preocupante, ya que la acción inadecuada en cuanto al manejo de residuos sólidos es recurrente, exponiendo así un desinterés para mantener los distintos espacios de la escuela totalmente limpios. Al preguntar el lugar donde termina la basura que producen en sus acciones cotidianas escolares, los estudiantes reconocieron que termina en la quebrada $50 \%$, quemada un $40 \%$ y $10 \%$ es arrojada a los vertederos (Gráfico 2). Esto demuestra una vez más, la existencia de prácticas ambientales inapropiadas producto de la poca formación en materia ambiental; los estudiantes no son conscientes de que arrojar residuos a la quebrada causa contaminación al recurso hídrico, lo que puede causar que muchas especies que allí habitan mueran, y además olvidan que la comunidad hace uso de este recurso natural para la toma no solo del preciado líquido sino de la oferta de peces que esta produce. 


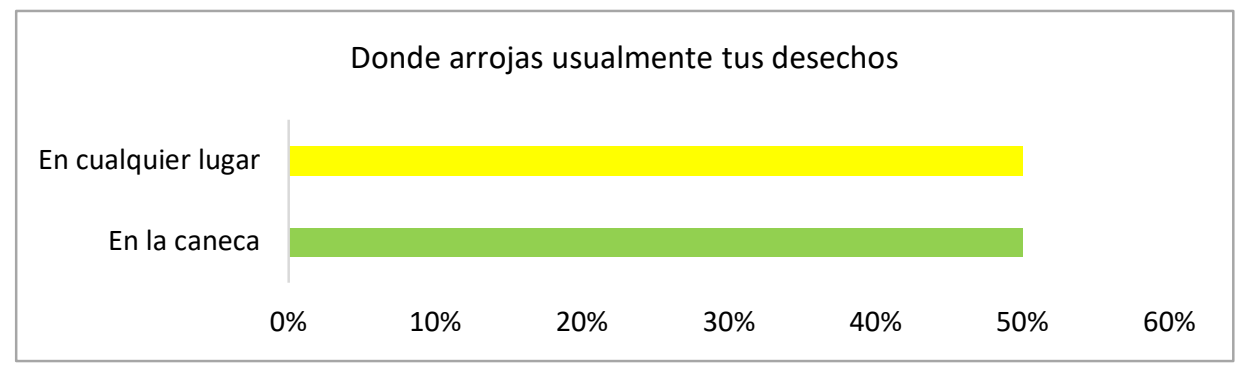

Gráfico 1. Destino de la basura en la escuela de acuerdo con los estudiantes. Fuente: Elaboración propia

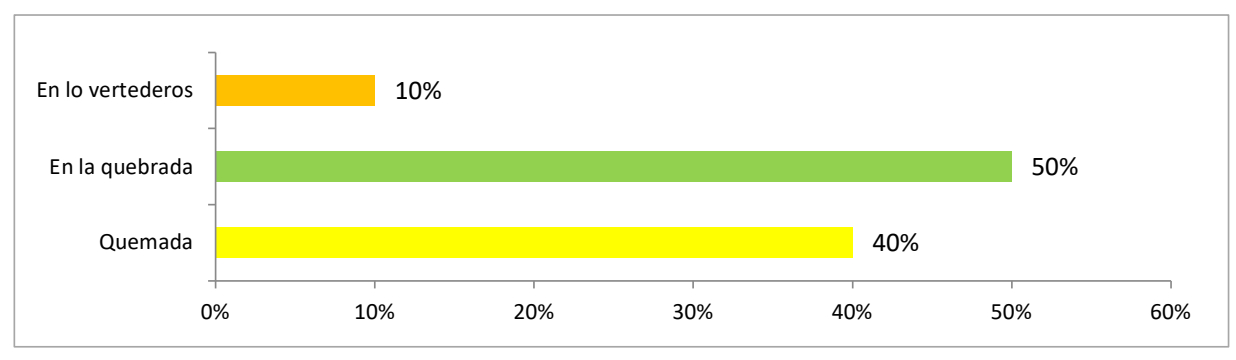

Gráfico 2. Percepción de los estudiantes sobre el destino final de los residuos que se producen en la institución.

Fuente: Elaboración propia.

Al preguntar a los docentes sobre el manejo que dan los estudiantes a las basuras producidas en la escuela respondieron que la gran mayoría de los educandos arroja los desechos en cualquier lugar, y que es común encontrar las aulas, el patio, las jardineras e incluso los baños colmados de residuos sólidos producto de las actividades diarias de los estudiantes de la institución. En conclusión, se puede decir que un alto porcentaje de los estudiantes no hace uso adecuado de las canecas puesto que arrojan los desechos en cualquier lugar, hábitos y comportamientos que reflejan prácticas ambientales negativas, corroborada por los docentes, estas acciones revelan en los y las jóvenes, poco sentido de pertenencia, a pesar de contar con recipientes para depositar los restos, producto de sus interacciones en la escuela, no lo hacen cotidianamente.

La observación participante confirmó los resultados anteriores, en tanto, se pudo exponer, que la cantidad de basura en los distintos espacios de la escuela va aumentando a medida que avanza la jornada escolar, situación que ocurre principalmente en el patio, durante el descanso. Al finalizar la jornada escolar la cantidad de desechos aumenta significativamente lo que denota actitudes, hábitos y comportamientos inadecuados con el ambiente. Asimismo, en los actos cívicos y culturales se observaron los espacios 
institucionales desaseados, basuras en el patio de la institución; al finalizar dichos actos se encontró que los niveles de desaseo son alarmantes, hay residuos en los distintos espacios de la institución, del mismo modo, se pudo determinar que los estudiantes no les dan uso a los contenedores, ya que generalmente están vacíos después de terminar este tipo de actividades lo que indica prácticas ambientales inadecuadas.

Manejo de energía. El manejo de energía resulta en este momento una acción que conlleva a la conservación del ambiente, y es necesario que sea constante, dado que, ayudaría a las generadoras en tiempos de sequía. Una visión más completa sería que el buen manejo de energía constituya un acto humano por el bienestar de la naturaleza que se vea manifestado en los comportamientos de todas las personas. En cuanto a las acciones que realizan los estudiantes para ahorrar energía, estos manifestaron que apagan ventiladores, los equipos de cómputo, bombillas y televisores; los docentes expresaron que los educandos no lo hacen de manera voluntaria, siempre depende de que ellos se las recuerden, lo cual fue corroborado con la guía de observación, pues la mayoría de los estudiantes no apagan bombillas y los que lo hacen, no lo hace por voluntad, sino que esperan que el docente les de la sugerencia, así mismo se estableció que en promedio la mitad de los educandos apaga los ventiladores. En este aspecto los estudiantes demuestran que son capaces de hacer un ahorro de energía voluntario, pero es preocupante el porcentaje que no lo hace.

Aseo y mantenimiento de áreas comunes. La escuela es un espacio en donde el estudiante tiene la oportunidad de compartir con sus pares experiencias académicas, deportivas, recreativas, personales y hasta familiares que de una u otra manera fortalecen su aprendizaje, sus lazos de amistad y compañerismo que tributan a su proceso de formación para interactuar en la sociedad en la cual debe desenvolverse. En cuanto al cuidado de jardines y áreas comunes los educandos expresan que lo hacen, algunas veces $57 \%$, casi siempre 17\%, siempre el 9\% y nunca el 17\% (Gráfico 3). Los datos anteriores, distan en buena medida de la realidad institucional dado que las observaciones realizadas dan cuenta de que los jardines de la escuela están descuidados muy pocos estudiantes los riegan, algunos dañan las plantas y las áreas comunes tales como patio, baños y lugares de recreo permanecen desaseadas, sumado a esto hay un buen número de educandos que no cumplen con los turnos de aseo que acordaron voluntariamente con sus compañeros y los docentes deben estar vigilantes para que estos acuerdos se cumplan; dando cuenta de actitudes, comportamientos y hábitos no favorables con el ambiente. 


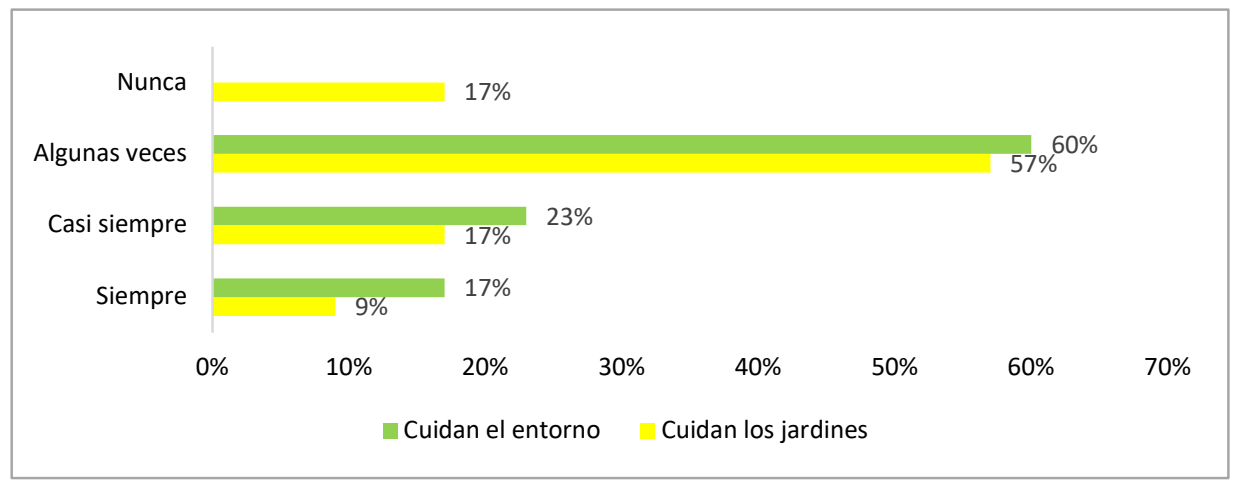

Gráfico 3. Cuidado del entorno escolar, jardines y áreas comunes por parte de los estudiantes.

Fuente: Elaboración propia.

Al preguntar a los estudiantes si se preocupan por el cuidado del entorno escolar y de los animales y plantas que hay en la institución, sólo un 9\% reconoce que siempre, mientras que la mayoría (60\%) algunas veces, siendo un resultado alarmante, y más cuando las observaciones hechas demuestran que el número de estudiantes que no se preocupan por esto es mayor. Dado el estado físico de la institución y los llamados de atención de los docentes por el no cuidado del entorno escolar, se suma a esto que las observaciones realizadas arrojaron como resultado que la gran mayoría de los educandos no respeta la fauna que vive o llega a la escuela lo que es más inquietante aún, pues estos son parte fundamental del ambiente, todo lo anterior refleja las malas prácticas ambientales de los estudiantes de la Institución Educativa Morindó Santa Fe.

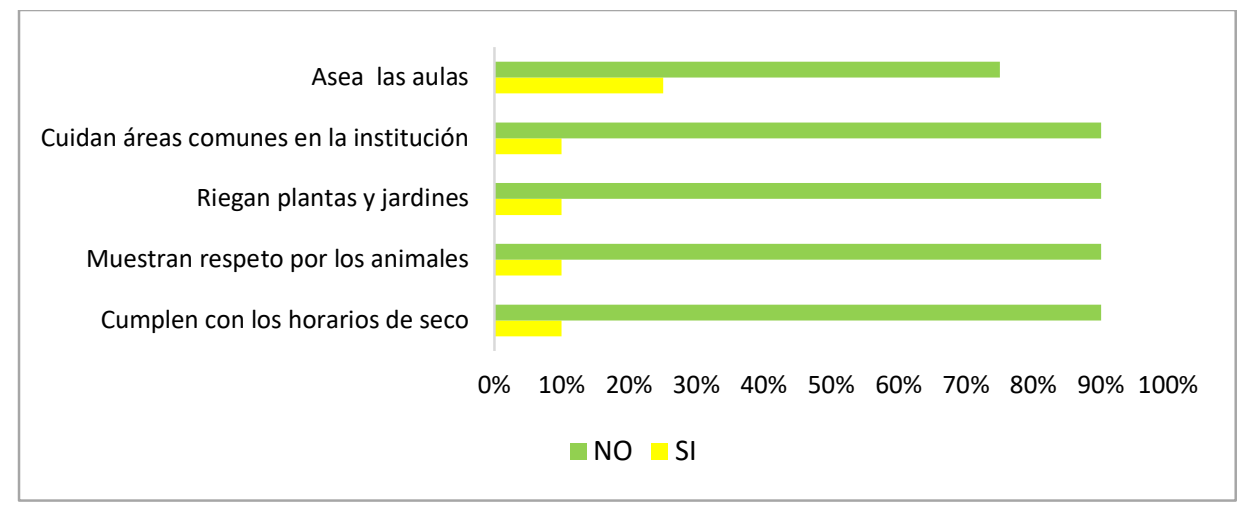

Gráfico 4. Cuidado de jardines, áreas comunes y cumplimiento de horario. Guía observación. Fuente: Elaboración propia.

Al analizar los datos recogidos a través de la guía de observación se encontraron resultados comunes en tanto que, el $75 \%$ no asean aulas; un $90 \%$ no riega plantas, no cuida jardines ni áreas comunes, otro 90\% no muestra respeto por los animales que llegan al entorno escolar y un porcentaje igual (90\%) no cumple los horarios de aseo, siendo un aspecto Revista Assensus | Vol. 5 | Núm. 9 | 2020 | 
negativo en sus prácticas ambientales (Gráfico 4). Los resultados producto de la observación dejan en evidencia que se necesita mayor trabajo en educación ambiental, pues los educandos expresan que tienen buenos hábitos, comportamientos y actitudes con el ambiente, pero las observaciones efectuadas no lo demuestran así.

EI PRAE y su dinamización. En este aspecto es importante mencionar que los educandos encuestados en la Institución Educativa Morindó Santa Fe, señalaron que la mayoría no conoce del PRAE, es decir, desconocen la existencia del proyecto, lo que lleva a pensar que este es un documento de papel, que se tiene solo para cumplir los requerimientos de la Secretaría de Educación, pero no para educar ambientalmente a los estudiantes, como debería darse.

Es importante destacar que una vez se defina, diseñe y plantee el PRAE, todos los miembros de la comunidad educativa (directivos, administrativos, docentes, estudiantes y padres de familia y personal de servicios generales), deben participar de manera colectiva en lo educativo -formativo desde la interdisciplinariedad y la transversalidad en las asignaturas, mediante trabajo comunitario, donde se evidencie la participación de todos los estamentos lo que posibilitaría una real formación en este ámbito, además su dinamización permitiría el desarrollo de capacidades que transformen las malas prácticas ambientales.

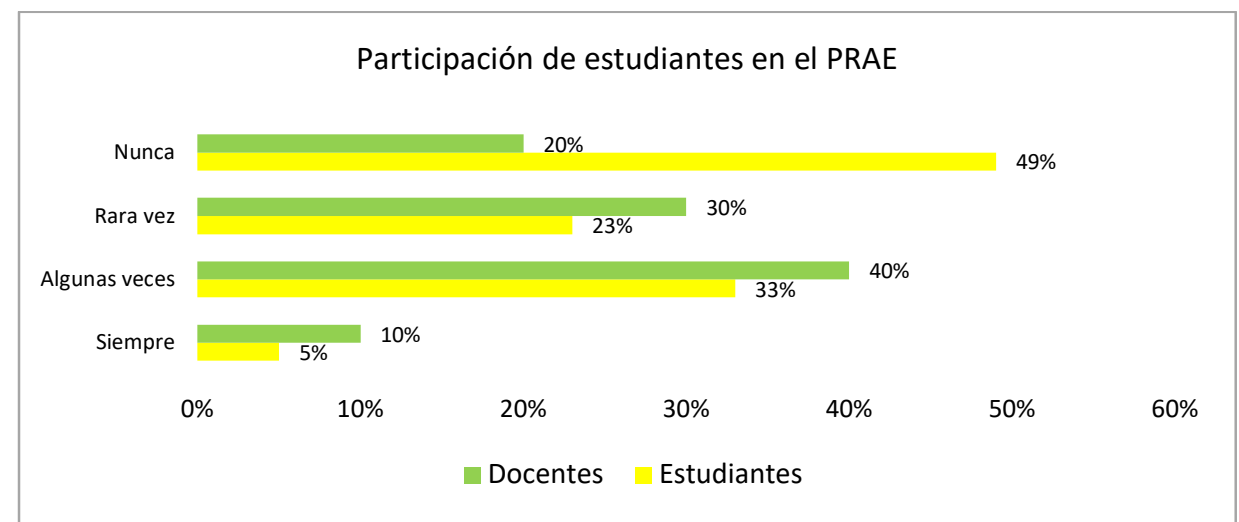

Gráfico 5. Participación de estudiantes en el PRAE, de acuerdo con estudiantes y docentes.

Fuente: Elaboración propia.

EI PRAE no se ha dinamizado, por ello los estudiantes manifestaron en un $40 \%$ que nunca participa en actividades relacionadas con el mismo, el resto (13\%) reconoce que rara vez participa, y el 33\% que algunas veces, lo que puede estar relacionado con la celebración de algunas fechas ambientales, información que a su vez fue confirmada por los docentes, al expresar que los estudiantes en un $20 \%$ no participan, rara vez $30 \%$, casi siempre $40 \%$ (Gráfico 5). 


\section{Discusión}

Las prácticas ambientales, deben ser consideradas cuando se diseñan e implementan programas de educación ambiental como respuesta a la responsabilidad de formar ciudadanos íntegros con alta valoración por el ambiente. Así, la educación ambiental es una alternativa para generar valores que se traducen en conductas y actitudes favorables para promover el desarrollo equilibrado en armonía con el entorno circundante; incrementando capacidades plenas en las diversas dimensiones del ser humano como la intelectual, moral y espiritual (Fuentealba, 2018, p. 168). Los resultados del presente estudio precisan, que existe poco conocimiento ambiental, por parte de los estudiantes, por lo que se infiere que se requiere materializar la educación ambiental en relación a aspectos ambientales relacionados con el manejo de recursos y el planteamiento de situaciones concretas a las realidades ambientales de la IE Morindó, este modo se incrementará el acervo cognitivo sobre el ambiente, lo cual normalmente tiene alta influencia en la conducta proambiental (Fuentealba, 2018; Alea, 2006). Al respecto, Sandoval (2012, p.190) indicó que no solo se debe enfatizar en la formación de conocimientos y actitudes proambientales en el diseño e implementación de programas de educación proambiental o sustentable, sino en el desarrollo de patrones de comportamiento acordes con los objetivos asociados con la protección y cuidado de este.

Con relación a los recursos, en la actualidad, existe un crecimiento amplio del consumo del agua, además de la degradación de su calidad en relación con vertimiento de desechos con residuos contaminantes producto de acciones cotidianas y de labores agrícolas y ganaderas, a un ritmo de asimilación superior al de los ecosistemas naturales, lo que implica un compromiso de muchos sectores económicos, políticos, educativos, e incluso el comunitario en tanto su protección y uso adecuado, requiere del compromiso de todos. El ser humano, en su afán de desarrollo, cada vez contamina más las fuentes de agua sin tener en cuenta la importancia de este recurso, y la Institución Educativa Morindó Santa Fe no es ajena a esta problemática, los estudiantes hacen mal uso del agua, tienen el hábito de arrojar las basuras a la quebrada Morindó que pasa cerca de la escuela, quizá porque ha sido una mala práctica que se ha mantenido por generaciones en los habitantes, quienes además aducen "que las lluvias arrastran las basuras en invierno, entonces esa es la forma más fácil de eliminarlas", esta manera de pensar y actuar no dimensionan el daño causado a los cuerpos acuíferos a la flora y fauna, acciones que dan cuenta de hábitos y comportamientos inadecuados con el ambiente, desconociendo que el agua es un recurso valioso, el cual requiere ser conservado y racionalmente utilizado, no se le ha dado la importancia y el valor que esta tiene, un buen número de educandos la desperdicia, pese a los llamados de atención que hacen los docentes para que hagan buen uso este recurso, pero sin importar las consecuencias que sus acciones pueden tener en la naturaleza los educandos no cuidan el este recurso. 
Ahora bien, en la zona rural donde se ubica la escuela, requiere un mayor esfuerzo conseguir este líquido, dado que no cuentan con acueducto y en la mayoría de los casos se transporta en bestias de carga o en hombros y son ellos mismos o sus parientes quienes tienen que hacer este trabajo. El desperdicio del agua en la institución lleva a su carencia, lo que a su vez limita el aseo diario de los baños, generando malos olores, además, el riego de las plantas del jardín, lo que causa deshidratación de estas, su deterioro y posterior muerte. Estas acciones convocan a la necesidad de aplicar estrategias que impacten en los educandos y los ayuden a transformar las prácticas ambientales inadecuadas, sensibilizarlos y lograr concientizarlos con relación al consumo responsable del agua, lo cual debe traducirse en buenas prácticas ambientales siendo necesaria una estrategia de educación que logre un equilibrio entre el individuo y la naturaleza, fomentando el desarrollo de las ciencias y la conciencia ambiental entre las nuevas generaciones (Fuentealba, 2014; Mendieta y Gutiérrez, 2014; Fuentealba, Marín Isamit, Castillo Retamal y Roco Fuentes 2017).

En cuanto a la producción y disposición de basuras la sociedad siempre ha generado desechos como resultado de los procesos de producción y consumo para satisfacer sus necesidades. Tarde o temprano los recursos extraídos de bosques, minas, pozos, mantos acuíferos, y la tierra misma se convierten en basuras, desperdicios o desechos (Medina, 1997, p.59). El buen manejo de las basuras es fundamental en cualquier espacio, en tanto, se relaciona con la existencia de buenas prácticas que favorecen el cuidado del ambiente. Es notorio, en las respuestas de estudiantes y docentes, y de las observaciones realizadas que los escolares tienen prácticas ambientales desfavorables, lo que es preocupante, ya que la acción inadecuada en cuanto al manejo de residuos sólidos es recurrente, pues no usan los contenedores, arrojan basura en los distintos lugares de la institución lo que evidencia un desinterés por mantener los distintos espacios de la escuela limpios y el desconocimiento de las afectaciones sobre la salud y la calidad del paisaje de este tipo de acciones por lo que hace falta un trabajo en formación de conocimientos, hábitos y comportamientos desde edades tempranas para que haya un cambio de actitud en el estudiantado con relación al manejo de desechos, así como lo descrito por Ángel Maya, (2003) se debe brindar herramientas necesarias para establecer vínculos éticos con la naturaleza, además la adquisición de una conciencia ambiental junto con actitudes de respeto hacia el ambiente, debe ser un proceso iniciado desde la infancia dado que permite la apropiación consciente de la apreciación de la naturaleza.

No existe una responsabilidad propia del estudiante por aportar al buen manejo de los residuos sólidos. Estas acciones o prácticas ambientales inapropiadas se pueden dar por falta de una orientación ambiental que se inicia en la casa y es continuada en las escuelas, y la no preocupación por los problemas del ambiente en los espacios escolares, resulta lamentable que la mayoría del estudiantado está siempre produciendo residuos sólidos, que no sabe cómo manejar, por lo que termina arrojándolos en cualquier lugar en la mayoría de los casos. Esto puede mejorarse si se fomentan campañas eco pedagógicas donde el estudiante 
interiorice que sus buenas prácticas ambientales son fundamentales para el mejoramiento del ambiente y su calidad de vida.

Otro aspecto que ya se mencionó es la contaminación de las fuentes de agua con residuos sólidos y otras sustancias cosa que demuestra una vez más, la existencia de prácticas ambientales inadecuadas, producto de la poca formación en materia ambiental; los estudiantes no son conscientes de que arrojar residuos a las quebradas causa una contaminación al recurso hídrico. Por lo que se hace necesaria la formación en conocimiento sobre el ambiente lo que ayudaría a formar conciencia de los impactos de las actividades antrópicas sobre los ecosistemas y sus efectos sobre el bienestar humano. A su vez, la conciencia ambiental conlleva a valorar los diferentes ecosistemas, lo que debería conducir a la adopción de una actitud y toma de acciones ecológicas. De la misma manera, existe un desconocimiento de los estudiantes del efecto de algunas prácticas ambientales, por ejemplo, al quemar la basura, se desprende humo que puede ocasionar enfermedades respiratorias y alérgicas, así como daño a la capa de ozono y al ambiente en general. En este mismo orden de ideas, las basuras en las quebradas o la quema de esta es una práctica ambiental inadecuada que es frecuentemente aplicada en su núcleo familiar y que ha sido la constante dentro de su comunidad. Por tal motivo, se hace necesario sensibilizar a los estudiantes sobre cuál debería ser la disposición final de cada residuo sólido que se produce en sus hogares y en la institución. Se puede decir que uno de los principales problemas ambientales que tiene la Institución Educativa Morindó Santa Fé, es el incorrecto manejo de residuos sólidos.

El buen manejo de la energía eléctrica hoy día es en una acción que conlleva a la conservación del ambiente, siendo un acto humano por el bienestar de la naturaleza. En este aspecto algunos estudiantes demuestran que son capaces de hacer un ahorro de energía voluntario, probablemente un buen hábito que traen de los hogares y que se extiende a la escuela. El ahorro de energía sería más significativo en la escuela si los estudiantes estuvieran atentos a los altos costos de las tarifas de energía en sus hogares y en la escuela, pero realmente un alto número no se interesan por ahorra energía. Según Tonello y Valladares, (2015) "la conservación de la energía es un importante subgrupo del comportamiento proambiental, sugiriéndose su abordaje desde políticas, acuerdos, programas educacionales, entre otros (p.47). Para finalizar se puede decir, que aunque el manejo de la energía es el problema ambiental de menor impacto en la institución educativa Morindó Santa Fe, esto no deja de ser un problema, dado que hay prácticas ambientales inadecuadas y que deben ser transformadas a través de un proceso de concienciación mediante una educación ambiental que conlleve a cambios en la forma de pensar de actuar y de entender los problemas ambientales por parte de los estudiantes, en este sentido, la educación entendida como agente de cambio y transformación social, adquiere un rol preponderante para generar y promover cambios en la esfera social, a través de la sensibilización, concientización y presentación de contenidos ambientales. 
Otro aspecto relevante, son los espacios de la escuela donde el estudiante comparte con sus compañeros, fortalecen su aprendizaje y sus lazos de amistad, un lugar de encuentro para los educandos, son las áreas comunes al interior de la escuela, los sitios de descanso en donde ellos juegan, dialogan y aprenden sobre diferentes temas, deben ser sitios agradables a los sentidos y cómodos, entonces, es necesario el cuidado de estos espacios, en cuanto al aseo, y el orden que deben ser esenciales para su adecuada utilización. Con relación al cuidado de las áreas comunes, riego de jardines, flora y fauna así como hacer el aseo, los estudiantes respondieron que la mayoría tienen buenos hábitos y comportamientos en el cuidado de las mismas, los docentes por su parte respondieron que aproximadamente la mitad realiza estas prácticas correctamente por lo dicho en las encuestas que respondieron, pero al comparar estos resultados con las observaciones efectuadas se encontró que la mayoría de los estudiantes tienen actitudes, hábitos y comportamientos inadecuados en el cuidado de las áreas comunes. Muchos de estos jóvenes no riegan jardines ni cuidan las plantas ni los animales que viven en el entorno escolar, ensucian las distintas zonas de la institución con residuos y casi no cumplen con los compromisos de aseo que adquirieron voluntariamente y es necesaria la intervención de los maestros para su cumplimiento.

Las respuestas dadas por los docentes muestran una notable falta de interés de los estudiantes con respecto al ambiente, es necesario destacar que cuando una persona se compromete a adquirir una actitud, suscita en su interior una fuerza eficaz para alcanzar ese objetivo. El compromiso es un salto cualitativo que le sitúa mucho más cerca de la meta propuesta. Asumiendo el compromiso son más permeables a la influencia configuradora de un valor proclamado como óptimo. Es notable la diferencia entre una disposición interior de responsabilidad y una disposición neutral. En este sentido, se requiere un proceso formativo en los estudiantes que genere cambios en esos comportamientos y que los lleve a asumir una actitud de compromiso, no solo con el entorno escolar, sino también con el familiar, llevándolos a transformar verdaderamente sus acciones negativas. Estos aspectos requieren de un cambio de conducta que perdure en el tiempo, el cual solo puede ser logrado a través de un proceso de concienciación, así mismo, se evidenció, que no existe el deber por parte del estudiantado a velar por el buen estado de estos lugares en la escuela. A través de las acciones formativas, se espera despertar el cuidado, y la sensibilidad ambiental del estudiante. De igual manera, se hace necesario crear espacios para la formación ambiental en donde cada uno de los estamentos se sienta comprometido con el cuidado, embellecimiento y preservación de las áreas comunes en aras de generar mayor proceso de motivación para compartir no solo el espacio físico de la escuela, sino también la interacción social, dado que docentes y estudiantes comparten más de la mitad de las horas del día.

A pesar de la existencia de los Proyectos Ambientales Escolares como estrategia para abordar la dimensión ambiental en la escuela, no se ha reflejado una proyección que permitan transformar las situaciones educativas que permitan visibilizar la acción ambiental en la transformación de las realidades ambientales (Flórez Restrepo 2013, p.131). Desde su 
promulgación, los PRAE, se han establecido como espacios de participación, en la cual toda la comunidad educativa debe desarrollar e implementar soluciones acordes con las dinámicas naturales y socioculturales del entorno donde se desarrollan. Este hace parte de un proceso de contextualización alrededor del cual se identifican problemáticas ambientales (naturales, sociales y culturales) para ser intervenidos a través de diferentes acciones (Velásquez, 2009, p.41). Sin embargo, en la IE Morindó Santa Fé, existe, pero no se ha dinamizado, no se transversaliza, y mucho menos se trabaja de manera interdisciplinaria y no se invita a toda la comunidad a ser parte activa en la ejecución de los proyectos asociados al mismo.

Es necesario el estudio sobre acciones que pueden predecir el comportamiento ambiental de las personas en determinado espacio, puesto que algunas de ellas optarán por mostrar prácticas favorables para el cuidado de todos los factores ambientales del entorno en que habitan, otros en cambio se inclinan por realizar acciones poco amigables con el ambiente como el uso poco racional de los recursos de la naturaleza. Aguilar (2006) destaca lo expresado por Berenguer y Martín, 2003), que el comportamiento se relaciona con el conjunto de variables personales como actitudes, valores, normas personales, creencias, aptitudes, expectativas propias, que unidas a otras de tipo contextual como el espacio físico y social definen el espacio vital de la persona y por lo tanto su predisposición hacia el medio físico, social y ambiental, es decir, se relacionan con las motivaciones que tienen las personas que median un tipo de conducta. (p.20) En este sentido, hay un desinterés del estudiantado por sentir que el cuidado de la naturaleza es fundamental para su existencia, no es consciente que vive en un mundo natural en donde la población debería en primer lugar cuidar, amar, proteger, y preocuparse por cada elemento que exista en el ambiente. Conservar la naturaleza no puede ser de unos pocos que se hacen llamar ecologistas, la razón esencial de la vida en la Tierra debe ser el uso racional de los recursos que provienen de la naturaleza, y en retribución el ser humano debe ser un ambientalista que vele por la protección de la misma, y sea un pedagogo en su entorno para que la tarea, por el bienestar de todo lo que lo rodea sea perdurable en el tiempo.

Es importante destacar que a partir de la industrialización y el privilegio que se le ha dado al desarrollo económico, como parte del capitalismo exacerbado, la humanidad se ha acostumbrado a ver la naturaleza como algo que está totalmente a nuestra disposición, un almacén de recursos del cuál extraer materias primas de provisión infinita, y un vertedero ilimitado donde depositar residuos, este tipo de creencias, actitudes y comportamientos y sus consecuencias desde hace décadas, ha dado origen a la "crisis ecológica" lo que demuestra que no se pueden mantener por más tiempo estas actitudes, sobre todo en la generación joven, lo que invita a un compromiso de los docentes en un proceso de formación ambiental que permita transformar al sujeto discente para lograr cambios en estas formas de actuar sobre la naturaleza. De acuerdo con Alea (2006 p.2) la educación ambiental, debe llevar a la formación de seres activos en la solución de los diversos problemas ambientales que enfrenta nuestro medio ambiente. Esto supone la asunción por parte de los mismos, de la 
responsabilidad con su propio destino, y cambios notables en pensamientos y actitudes que faciliten nuevas concepciones del mundo.

Lo anteriormente descrito, da cuenta de la necesidad de un proceso de formación inmerso en el PRAE, que lleve a los estudiantes de la Institución Educativa Morindó Santa Fe a transformar sus actitudes, hábitos y comportamientos, a generar en ellos un sentido de respeto y de valoración de la naturaleza, propender por encontrar, individual y colectivamente otro estilo de vida, y el reconocimiento de la condición sistémica de la naturaleza. De acuerdo con Jaimes y Barreto (2015). el comportamiento proambiental debe formar parte de la rutina de las personas, aprendido en diversas áreas de la vida e incorporado en sus hábitos (p.731). Se requiere generar en los estudiantes conductas proambientales, las cuales generen "acciones intencionales y efectivas que resultan en la conservación del ambiente, constituyendo uno de los componentes clave en la conformación de la conducta sustentable (Tonello y Valladares, 2015, p.47).

\section{Conclusiones}

La caracterización de las prácticas ambientales evidenció acciones negativas o desfavorables en un alto porcentaje de los miembros de la comunidad estudiantil, quienes realizan un manejo inadecuado del recurso agua en la escuela. En cuanto al manejo de residuos sólidos que es quizá el principal problema ambiental de la institución, los estudiantes arrojan papel, bolsas, plásticos y otros desechos en cualquier parte. Durante los actos culturales y cívicos la producción de desechos aumenta considerablemente y el uso de las canecas es casi nulo lo que denota prácticas inapropiadas hacia el ambiente. También se encontró un significativo número de educandos que no hacen un uso racional de energía eléctrica, común encontrar ventiladores y bombillas encendías sin que se estén usando, al igual se encontraron equipos de cómputo encendidos o conectados después de las clases e incluso cargadores de celulares dejados en los tomacorrientes de los salones.

Es notorio en los hallazgos enunciados en las líneas anteriores, que el desconocimiento de la problemática ambiental que hoy sucede en el mundo y de conductas que van en contravía del cuidado y protección del ambiente no son ajenos a la realidad de la Institución Educativa Morindó Santa Fé, pues se están dando prácticas ambientales inadecuadas, por lo que se requiere implementar estrategias de transformación de comportamientos, hábitos y actitudes. La escuela a través de sus procesos formativos integrales debe aportar al fortalecimiento de la concienciación ambiental, de ahí que los comportamientos, las creencias, los hábitos deben ser evaluados para diseñar estrategias metodológicas, pedagógicas y didácticas que permitan formar estudiantes críticos, reflexivos que se proyecten a la sociedad con acciones proambientales en el aula y fuera de ella hacia el 
ambiente para lograr una mejor calidad de vida por lo que es necesario dinamizar el PRAE y que este operativamente articulado al Proyecto Educativo Institucional (PEI) desde una perspectiva integral, pueda promover la formación de un estudiantado que propenda por prácticas ambientales amigables con su entorno, es decir, ciudadanos responsables con el ambiente.

\section{Referencias}

Aguilar, M (2006). Predicción de la conducta de reciclaje a partir de la teoría de la conducta planificada y desde el modelo del valor, normas y creencias hacia el medio ambiente. Tesis doctoral. Universidad de Granada. España

Alea, A. (2006). Diagnóstico y potenciación de la Educación Ambiental en jóvenes universitarios. Odiseo, Revista electrónica de Pedagogía. Año 3, n 6, 1-29.

Boff, L., y Valverde, J. (2002). El cuidado esencial ética de lo humano, compasión por la tierra. Madrid, España: Trota.

Calderón, R., Sumarán, R., Chumpitaz, J., y Campos, J. (2011). Educación Ambiental. Aplicando el enfoque ambiental hacia una educación para el desarrollo sostenible. Perú, Gráficos Kike 213 p.

Carabias Barceló, V, (2002). Conciencia ambiental y comportamiento ecológico un análisis de la escala GEB (General Ecological Behavior) de Kaiser. Revista Internacional de Sociología. Vol 60 (33), pp. $133-170$

Castillo, F., y Fuentealba, M. (2014). Compendio de buenas prácticas ambientales. Talca: Universidad Católica del Maule

Castillo -Retamal, F., y Cordero Tapia, F. (2019). La educación ambiental en la formación de profesores. Revista UCMaule $N^{\circ} 56, \mathrm{pp}, 9-28$.

Cuartas-Gómez, E.; Palacio-Duque, A.; Ríos-Osorio, L.A.; Cardona-Arias, J.A.; Salas-Zapata, W.A. (2019). Conocimientos actitudes y prácticas (CAP) sobre sostenibilidad en estudiantes de una universidad pública colombiana. Rev. U.D.C.A Act. \& Div. Cient. 22(2) 385.

De Souza Minayo, M. C. (2009). La artesanía de la investigación cualitativa. Buenos Aires: Editorial Lugar S.A 332p.

Ferrer, B.; Menéndez, L., y Gutiérrez, M. (2004). La cultura ambiental por un desarrollo sano y sostenible. La experiencia de Cayo Granma. Revista Electrónica. 59-79

Flórez, G. (2013). La dimensión ambiental en los escenarios escolares. Educación y Ciencia, (16). 129 - 146.

Fuentealba, M. (2014). Actitudes de estudiantes universitarios de la Universidad Católica del Maule frente a temáticas ambientales. Paideia, (55), 119-135. Recuperado de http://www.revistapaideia.cl/ 
Fuentealba, M. (2018). Valoración actitudinal proambiental: un análisis global en estudiantes de enseñanza primaria, secundaria y terciaria. Revista Luna Azul. No. 47. 159- 176. DOI:10.17151/luaz.2019.47.9.

Fuentealba Cruz, M., Marín Isamit, F., Castillo Retamal, F., y Roco Fuentes, L (2017). Análisis de la experiencia pedagógica: Campamento EXPLORA Chile VA. Valorando la Biodiversidad Maulina. Revista Electrónica “Actualidades Investigativas en Educación, Volumen 17 Número 1 pp. 1-25

Galli, F., Bolzan de Campos, C., Bedim, L., y Castellá, J. (2013). Actitudes hacia el medio ambiente en la infancia: un análisis de niños del sur de Brasil. Revista Latinoamericana de Psicología (45), 461-473

García, L. y Restrepo, A. C. (2015). Desarrollo humano y social en las prácticas ambientales de los graduados de la Maestría en Desarrollo Sostenible y Medio ambiente, Universidad de Manizales. Revista Virtual Universidad Católica del Norte, 44, 253-266. Recuperado de http://revistavirtual.ucn.edu.co/index.php/RevistaUCN/article/view/628/1163

Gómez, M. (2018). Educación para el desarrollo sostenible. Una mirada a los proyectos ambientales escolares PRAE. Libre Empresa, 15(2), 179-194.

Gomera, A., Villamandos, F. y Vaquero, M. (2013). Construcción de indicadores de creencias ambientales a partir de la Escala NEP [Construction of indicators of environmental beliefs from the NEP scale]. Acción Psicológica, 10(1), 149-160. http://dx.doi.org/10.5944/ap.10.1.7041

Gutiérrez, L. (2015). Problemática de la educación ambiental en las instituciones educativas. Revista Científica, 23, 57-76.

Jaimes, F y Barreto, C (2015). Estrategia Socio Educativa para el cuidado de lo público - mobiliario escolar- en el colegio Tomás Carrasquilla IED de Bogotá D.C. Bio-grafía Escritos sobre la biología y su enseñanza, 729-743.

Medina, M. (1997). Manejo de desechos sólidos y desarrollo sustentable. Económicas CUC, 25(1), 5971.

Mendieta, M y Gutiérrez, G. (2014). Actitudes ambientales hacia el agua, una exploración en estudiantes del Municipio de Ventaquemada (Boyacá). Luna Azul, (39), 40-62. Recuperado de http://www.scielo.org.co/scielo.php?script=sci_arttext\&pid=S1909-24742014000200004

Mora-Ortiz, J. (2015). Los Proyectos Ambientales Escolares. Herramientas de gestión ambiental. Revista Bitácora Urbano Territorial, 25(2), 67-74.

Novo, M. (2009). La Educación Ambiental, una genuina educación para el desarrollo sostenible. Revista de educación número extraordinario 2009, pp 195-217 Madrid, España.

Orbes, D., y Delgado, L. (2017). Fortaleciendo la enseñanza del ambiente a partir de las concepciones y prácticas ambientales de docentes y estudiantes del grado 7-1 de la I.E.M. Nuestra Señora de Guadalupe. Tesis de maestría, de la Universidad Santo Tomas de San Juan de Pasto. 
Pérez, H. (2015). Sentidos y prácticas ambientales en la Institución Educativa Cámara Junior de Armenia Quindío. Universidad de Manizales, Colombia.

Pérez-Vásquez, N. (2020). Educación Ambiental para docentes en formación de la Universidad de Córdoba- Colombia: hacia un ejercicio profesional apoyado en los principios de la sustentabilidad y la responsabilidad ética, política y social. Tesis Doctoral. Universidad Surcolombiana. Doctorado en Educación y Cultura Ambiental. 230 p.

Pineda, R., y Pinto, L (2018). Estrategias didácticas en educación ambiental para el fortalecimiento de buenas prácticas ambientales (Master's thesis, Escuela de Educación y Pedagogía). Universidad Pontificia Bolivariana, Leticia, Amazonas.

Presidencia de la Republica de Colombia (2016). Lineamientos de buenas prácticas ambientales.

Rengifo, B., Quitiaquez, L., y Mora, F. (2012). La Educación Ambiental una estrategia pedagógica que contribuye a la solución de la problemática ambiental en Colombia. XII Coloquio internacional de Geocrítica.

Rivera-Torres, P., y Garcés-Ayerbe, C. (2018). Desarrollo del comportamiento proambiental en los individuos y sus determinantes. Reis: Revista española de investigaciones sociológicas, (163), 59-78.

Sandoval, M. (2012). Comportamiento sustentable y educación ambiental: una visión desde las prácticas culturales. Revista Latinoamericana de Psicología. Volumen 44, número 1, p. 181-196).

Sabino, C. (1992). El proceso de la investigación. Caracas. Editorial Panamo.

SECRETARÍA DE AMBIENTE. ALCALDÍA DE QUITO. (2018). Manual de buenas prácticas ambientales para instituciones educativas.

SENADO DE LA REPÚBLICA DE COLOMBIA. DIRECCIÓN GENERAL ADMINISTRATIVA. (2011). Manual de Buenas Prácticas Ambientales. Bogotá, Colombia

Severiche-Sierra, C., Gómez-Bustamante, E., y Jaimes-Morales, J. (2016). La educación ambiental como base cultural y estrategia para el desarrollo sostenible. Revista TELOS. Vol. 18 (2), 266 281.

Tonello, G., y Valladares, N. (2015). Conciencia ambiental y conducta sustentable relacionada con el uso de energía para iluminación. Gestión y ambiente, 18(1), 45-59.

Universidad de Cádiz. Estudio diagnóstico ambiental UCA. Informe sobre buenas y malas prácticas ambientales en la UCA. 2010.

Velásquez J. (2009). La transversalidad como posibilidad curricular desde la Educación Ambiental. Revista Latinoamericana de Estudios Educativos 5(2), 29-44.

Vilches, A., y Pérez, D. (2016). La transición a la Sostenibilidad como objetivo urgente para la superación de la crisis sistémica actual. Revista Eureka sobre Enseñanza y Divulgación de las Ciencias, 13(2), 395-407. 